INPLASY

PROTOCOL

To cite: Ge et al. The efficacy and safety of Chinese patent medicine LWWL combined with nucleoside/nucleotide analogues in treatment of chronic hepatitis $B$ : A systematic review and metaanalysis. Inplasy protocol 2021100106. doi:

10.37766/inplasy2021.10.0106

Received: 27 October 2021

Published: 27 October 2021

Corresponding author: Yan Liu

liuyan5360@163.com

Author Affiliation:

Department of Infectious

Diseases, The Fifth Medical

Center of Chinese PLA General

Hospital.

Support: China Nation Natural Science.

Review Stage at time of this submission: Other - Not described (Ongoing).

\section{The efficacy and safety of Chinese patent medicine LWWL combined with nucleoside/nucleotide analogues in treatment of chronic hepatitis B: A systematic review and meta-analysis}

Ge, F1; Yang, Y2; Si, L3; Li, Y4; Li, L5; Liao, H6; Wang, J7; Zhang, S8; Sun, Q9; Xiao, $\mathrm{X}^{10}$; Liu, $\mathrm{Y}^{11}$.

Review question / Objective: This study aimed to assess the efficacy and safety of Chinese patent medicine Liuweiwuling tablet (LWWL) combined with Nucleoside/nucleotide analogues (NAs) in treating chronic hepatitis B (CHB) by metaanalysis.

Eligibility criteria: (1) The original data were published literature, which included randomized controlled trial (RCT) on LWWL combined with NAs (TAF, TDF, ETV, ADV, LdT and LAM) in the treatment of CHB. (2) Patients diagnosed with CHB according to clinical diagnostic criteria were included. (3) In terms of treatment measures, the control group was only treated with TAF, TDF, ETV, ADV, LdT and LAM, while the experimental group was treated with LWWL in the basis of control group. (4) Outcome indicators should include one or more of HBV DNA negative conversion rate, HBeAg negative conversion rate, ALT and AST. (5) Treatment lasted at least 12 weeks.

INPLASY registration number: This protocol was registered with the International Platform of Registered Systematic Review and Meta-Analysis Protocols (INPLASY) on 27 October 2021 and was last updated on 27 October 2021 (registration number INPLASY2021100106).

Conflicts of interest:

None declared.

\section{INTRODUCTION}

Review question / Objective: This study aimed to assess the efficacy and safety of Chinese patent medicine Liuweiwuling tablet (LWWL) combined with Nucleoside/ nucleotide analogues (NAs) in treating chronic hepatitis B (CHB) by meta-analysis.

Condition being studied: Hepatitis B virus (HBV) infection is still one of the serious public health problems in the world. It can 
increase occurrence risk of liver cirrhosis and hepatocellular carcinoma (HCC). An estimated 257 million individuals live with HBsAg positive, leading to more than 887000 deaths annually (Revill et al., 2019). HBV infection not only seriously harms the health of patients, but also increases the heavy medical burden. Interferon (IFN) and nucleoside/nucleotide analogues (NAs) as the first line drugs, have brought great benefit for patients especially in HBV DNA suppression. However, HBV is hardly to be eliminated from CHB patients, which is a major and huge challenge. In addition, HBV drug-resistance and adverse drug reactions (ADRs) are also significant factors influencing therapeutic efficacy. Great efforts have been made in developing more effective medicines and therapies in an attempt to increase clinical cure of CHB. Nowadays, there is an increasing interest in using complementary and alternative medicine combined with NAs, especially traditional Chinese medicine (TCM), for the treatment of CHB. Liuweiwuling tablet (LWWL) (approval number: Z20060238) is a drug approved by the China National Medical Products Administration (CNMPA) included in the national health insurance. It has good efficacy on anti-inflammation of chronic HBV infection. In addition, there are also some clinical reports that LWWL combined with NAs could improve the rate of HBV DNA undetectability and $\mathrm{HBeAg}$ compared to NAs used alone. Therefore, the study aims to evaluate the efficacy and safety of LWWL combined with NAs in the treatment of $\mathrm{CHB}$ through meta-analysis.

\section{METHODS}

Participant or population: Chronic hepatitis B (CHB) patients.

Intervention: LWWL+NAs.

\section{Comparator: NAs.}

Study designs to be included: The original data were published literature, which included randomized controlled trial (RCT) on LWWL combined with NAs (TAF, TDF, ETV, ADV, LdT and LAM) in the treatment of CHB.
Eligibility criteria: (1) The original data were published literature, which included randomized controlled trial (RCT) on LWWL combined with NAs (TAF, TDF, ETV, ADV, LdT and LAM) in the treatment of CHB. (2) Patients diagnosed with $\mathrm{CHB}$ according to clinical diagnostic criteria were included. (3) In terms of treatment measures, the control group was only treated with TAF, TDF, ETV, ADV, LdT and LAM, while the experimental group was treated with LWWL in the basis of control group. (4) Outcome indicators should include one or more of HBV DNA negative conversion rate, $\mathrm{HBeAg}$ negative conversion rate, ALT and AST. (5) Treatment lasted at least 12 weeks.

Information sources: The retrieval sources include PubMed, Chinese Scienceand Technology Journal Database (VIP), China National Knowledge Infrastructure (CNKI) and WanFang Data, China Bio Medical Literature (CBM), Web of Science, Cochrane library.

Main outcome(s): Outcome indicators should include one or more of HBV DNA negative conversion rate, $\mathrm{HBeAg}$ negative conversion rate, ALT and AST.

Quality assessment / Risk of bias analysis: We used the Cochrane Risk of bias assessment tool in the software of Review Manager 5.3 for RCTs to evaluate the quality of the included literature. According to its guidance, each domain of individual studies was graded as low, unclear, or high risk of bias.

Strategy of data synthesis: Heterogeneity among the included studies was checked by using the chi-square test and 12 test. 12 was used to quantify the heterogeneity. If 12 $\leq 50$, that suggests the not existence of heterogeneity, the fixed effect model would be used to combine data, otherwise, it would be random effect model.

Subgroup analysis: subgroup analyses between the LWWL+ETV/ADV/LAM/LdT and ETV/ADV/LAM/LdT groups were carried out. 
Sensitivity analysis: Sensitivity analysis performed by excluding low-quality literature.

Country(ies) involved: China.

Keywords: Chinese patent medicine; nucleoside/nucleotide analogues; hepatitis B virus; meta-analysis; randomized controlled trials.

Contributions of each author:

Author 1 - Feilin Ge.

Email: 1439312084@qq.com

Author 2 - Yan Yang.

Email: 670408459@qq.com

Author 3 - Lanlan Si.

Email: sll1900@163.com

Author 4 - Yuanhua Li.

Email: 1507965212@qq.com

Author 5 - Le Li.

Email: lilencd@126.com

Author 6 - Hao Liao.

Email: liaohao2011made@163.com

Author 7 - Jun Wang.

Email: wangjun00302@163.com

Author 8 - Shan Zhang.

Email: 2011210689@bjmu.edu.cn

Author 9 - Qin Sun.

Email: zxyjhsq@swmu.edu.cn

Author 10 - Xiaohe Xiao.

Email: pharmacy302xxh@126.com

Author 11 - Yan Liu.

Email: liuyan5360@163.com 